RECEPTOR SCP

NEW YORK-A new generation of
recombinant microbial screens will enable pharmaceutical companies to design assays not simply for binding but for function as well, which could mean substantial savings in drug screening programs. The most recent development is a yeast-based screen for steroid analogues, reported Donald McDonnell of Baylor College of Medicine (Houston, TX) at the American Society for Microbiology Conference on Biotechnology held here in late June.

Many diseases are linked with a particular membrane-bound receptor. In fact, four of the top selling drugs-ranitidine, cimetidine, propranolol, and salbutalmol-are receptor-selective. Traditional pharmaceutical screens use tissues rich in a particular receptor to detect binding. In recombinant surrogate systems, the assay can be improved in efficiency by providing pure populations of receptors. For instance, Janssen Pharmaceuticals (Beerse, Belgium), has been using Escherichia coli to express beta-receptor genes for nearly three years. According to Janssen's Jose Leysen, the system is rapid and cheap. "A single four-hour culture provides sufficient material to screen 70 drugs per week for two years," says Leysen.

Most of the receptors of current therapeutic interest belong to the $G$ protein-coupled receptor superfamily. $G$ proteins consist of three subunits: alpha, beta, and gamma.
PEENS DETECT BIO After binding an agonist, a receptor interacts with its membrane-associated $\mathrm{G}$ protein, facilitating dissociation of the $G$ protein into two components, alpha and beta-gamma. The alpha subunit, but not the betagamma, varies between receptor types. In a functional screen, elements of this signal-transduction pathway need to be expressed along with the receptor itself. Three distinct approaches are being taken to achieve this.

The first approach, pioneered by Donny Strosberg at the Institut Pasteur (Paris), is to provide $E$. coli with a suitable transduction and reporter system. This involves co-expression of the receptor gene and genes for the alpha, beta, and gamma G-protein subunits.

The second approach uses mammalian cells, which already possess the required G-protein apparatus, to express receptor genes. Tom Bonner's group at the U.S. National Institutes of Health (Bethesda, MD) has been at the center of these efforts and has supplied a number of drug companies with functional receptor expression in mammalian cell lines. These mammalian cells are relatively difficult to cultivate and yield low expression levels, two problems that will probably limit the use of such systems in screening.

The third route, using a Saccharomyces cerevisiae expression system, is, perhaps, a halfway house between the other two. S. cerevisiae has endogenous
BIOACTIVITY

G proteins, but they lack the specific mammalian alpha subunit needed for signal transduction. Therefore, King, et al. (Science 250: 121-123, 1990) cotransfected genes for the human beta 2-adrenergic receptor and the mammalian G-protein alpha subunit into the yeast. They could demonstrate effective coupling between the receptor, the alpha subunit, and the endogenous beta-gamma subunit. By linking the receptor-G-protein system to a beta-galactosidase reporter gene, they produced a screen for beta2adrenoceptor agonists which can be monitored colorimetrically and adapted to automation and highthroughput.

Baylor College's McDonnell has extended this approach by exploiting similarities between yeast and mammalian transcription activators. Mammalian steroid receptors are members of a superfamily of transcriptional activators. By expressing the soluble steroid-receptor gene in yeast, McDonnell has been able to reconstitute a steroid-dependent transcription activation system. The transcription system is linked to the essential URA-3 gene to provide an assay the endpoint of which is growth of yeast. McDonnell says that the system in various forms can be used to look for analogues or agonists of thyroid hormones, estrogen, or progesterone. Applications in the search for therapeutics for breast cancer and osteoporosis are already being explored.

-Alison Abbott

\title{
GENE THERAPY FOR AIDS?
}

FLORENCE-With CD4 receptor variants apparently floundering as therapeutic agents for AIDS, it was inevitable perhaps that the mechanisms by which HIV enters T lymphocytes would be reexamined. Speaking at the International Conference on AIDS held here in June, Robin Weiss from the Institute of Cancer Research (ICR, London) has now proposed that the virus may need to interact with a "second" unidentified molecule on the cell surface in order to penetrate the cell. "We have an idea," Weiss said, "that the second molecule could be a protease that cuts the envelope's gp120."

It was at ICR that much of the work that led to the identification of CD4 as a target molecule on the surface of particular types of cells infected by the virus was performed. The need for a second molecule may explain, said Weiss, why HIV cannot infect transgenic animals which express CD4. It might also explain the finding reported by Cecilia Cheng-Mayer of University of California School of Medicine (San Francisco) that different HIV strains seem able to infect different types of cells. Different HIV strains could identify different secondary molecules or recognize different parts of a single secondary molecule.

The idea of a second molecule is beguiling but not persuasive, according to William A. Haseltine at the Division of Human Retrovirology at the Dana Farber Cancer Institute (Boston, MA). "We believe that a nonspecific sticky attachment can occur, and for some cells this is sticky enough to trigger the fusion event," says Haseltine. "What we need to study is the functional envelope, the protein and local conditions on the cell membrane." His group is looking at the binding kinetics and thermodynamics of CD4 and gp 120. They think that binding is a two-step or threestep process. The initial binding step occurs rapidly at low temperature. The second step is an energy-dependent process resulting in a high affinity binding. The final step may be the formation of a microcrystaline structure of gp120 associated with CD4. "We believe that one of the reasons that infection with HIV through CD4 in chimpanzees does not proceed readily is that this microcrystalization does not occur," says Haseltine.

In another session in Florence, Haseltine was among those advocating gene therapy for the treatment of AIDS. He was joined by Robert Gallo of the U.S. National Cancer Institute (Bethesda, MD), although the ap- 